\title{
Donor nephrectomy of a patient with situs inversus totalis
}

\author{
Steve R Siegal* and Randy S Haluck
}

Fellow - Minimally Invasive Surgery, Penn State Hershey Medical Center, Hershey, Pennsylvania, USA

\section{Introduction}

Situs Inversus Totalis (SIT) is a rare entity of complete transposition of the abdominal and thoracic organs. The reported incidence of SIT varies between 1:8,000 and 1:20,000 [1]. This condition is often discovered incidentally as patients are generally asymptomatic. Historically, SIT was considered a contraindication to donation of the liver and heart for transplantation [2]. We report a case of living donor nephrectomy in a patient with SIT.

\section{Case Report}

A 35-year-old female with a history of hypothyroidism entered evaluation for living kidney donor in August of 2019 and was deemed an appropriate candidate. Her 24-hour creatinine clearance was $117 \mathrm{ml} /$ min and serum value of $0.88 \mathrm{mg} / \mathrm{dL}$ with a glomerular filtration rate of $>$ $60 \mathrm{ml} / \mathrm{min}$. Preoperative protocol included a CT angiogram to evaluate kidney and vasculature anatomy where SIT was diagnosed (Figure 1). Traditionally, the left kidney is used due to longer blood vessels, however given SIT a laparoscopic right donor nephrectomy was planned (Figure 2 ). The patient was placed in left lateral positioning and the laparoscopic ports were placed in the right upper quadrant, upper midline and right lateral quadrant. The splenic flexure was reflected medially to expose the anterior right kidney (Figure 3). The ureter was identified and isolated. The renal hilum was then exposed, and the artery and vein dissected and divided as well as the gonadal vein. The right kidney was mobilized and removed through a Pfannenstiel incision and prepared on a back table for donation. The patient made voluminous urine output, was awoken and taken to recovery. She recovered well and was discharged on postoperative day two. Three weeks later she was seen in clinic and doing well.

\section{Discussion}

SIT was once a contraindication to organ transplantation due to concern of visceral and vascular abnormalities. Despite previous hesitancy with liver and heart transplantation, many successful

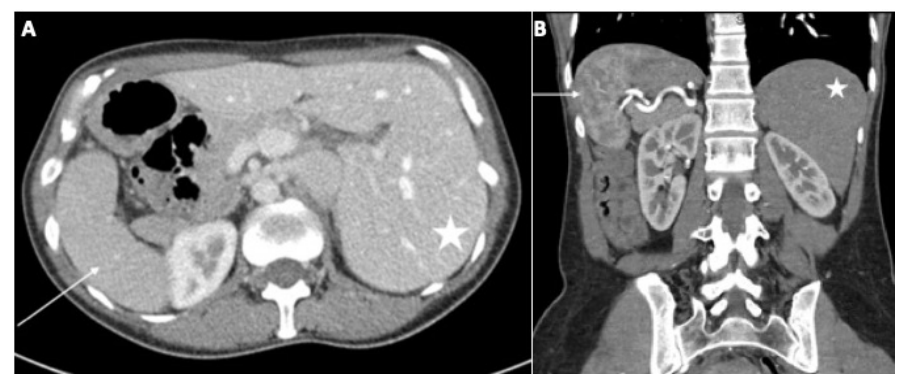

Figure 1. CT Images of the patient with SIT. A) Axial image showing the liver (star) on the patient's left and the spleen (arrow) on the patient's right. B) Coronal image showing the liver (star) on the patient's left and the spleen with its torturous artery (arrow) on the patient's right

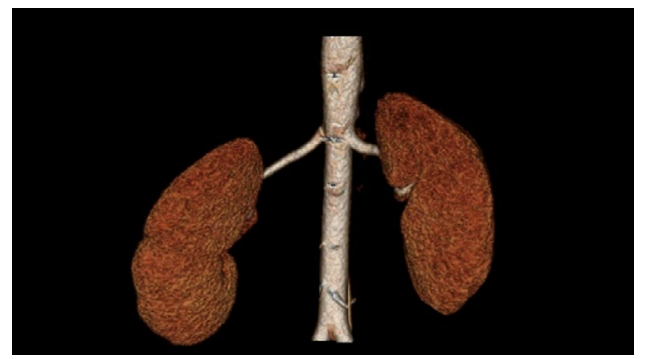

Figure 2. 3D reconstruction of CT angiogram showing kidneys and their arteries. This demonstrates a longer renal artery on the right

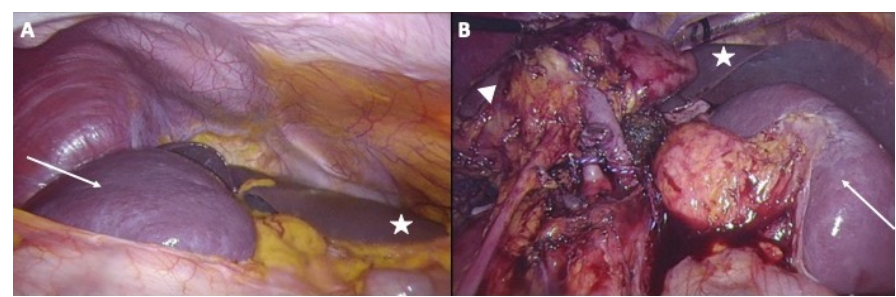

Figure 3. Intraoperative photographs. A) A view of the patient's right upper quadran with the spleen (arrow) occupying the upper quadrant and the liver (star) in the midline emanating from the left upper quadrant. B) The right kidney is dissected (arrowhead) with the renal vein ligated anterior to the artery. The liver again is shown (star). Note the right sided spleen (arrow) is reflected medially with the tail of the pancreas visible in the splenic hilum

transplantations in SIT patients have been described [3,4]. Kidney donation in patients with SIT has shown to be reliable in limited reports. Initial publications included kidneys from cadavers and organs procured with an open nephrectomy technique. In recent times, a minimally invasive approach to nephrectomy would be preferable. Similarly, living donation may lead to better patient or graft outcomes.

We present a case of successful laparoscopic living donor nephrectomy in a patient with SIT. There were no notable complications in the donor or recipient. What is important to consider in these cases is a thorough preoperative workup. In addition to determining appropriate candidacy for kidney donation, surgical factors such as organ size and vasculature must be studied. SIT donors should get a CT angiography study of their abdomen prior to donation to determine adequate renal vessel length, knowing that more than likely the right kidney would have a longer vessel as it approaches a left sided inferior vena cava. SIT,

${ }^{\star}$ Correspondence to: Steve R Siegal, Fellow - Minimally Invasive Surgery, Penn State Hershey Medical Center, Hershey, Pennsylvania, USA, E-mail: ssiegal@pennstatehealth.psu.edu

Received: February 27, 2020; Accepted: March 05, 2020; Published: March 10, 2020 
being an asymptomatic condition, should not put donors at increased risk of perioperative complications as long as preoperative planning by a skilled and experienced transplant surgeon is thorough.

\section{References}

1. Borgaonkar VD, Deshpande SS, Kulkarni VV (2011) Laparoscopic cholecystectomy and appendicectomy in situs inversus totalis: A case report and review of literature. $J$ Minim Access Surg 7: 242-245. [Crossref]
2. Polak WG, Chudoba PJ, Patrzalek D, Szyber P (2006) Organ donor with complete situs inversus. Case report and review of the literature. Ann Transplant 11: 43-46. [Crossref]

3. Raynor SC, Wood RP, Spanta AD, Shaw BW (1988) Liver transplantation in a patient with abdominal situs inversus. Transplantation 45: 661-663. [Crossref]

4. Doty DB, Renlund DG, Caputo GR, Burton NA, Jones KW (1990) Cardiac transplantation in situs inversus. J Thorac Cardiovasc Surg 99: 493-499. [Crossref]

Copyright: $\odot 2020$ Siegal SR. This is an open-access article distributed under the terms of the Creative Commons Attribution License, which permits unrestricted use, distribution, and reproduction in any medium, provided the original author and source are credited. 